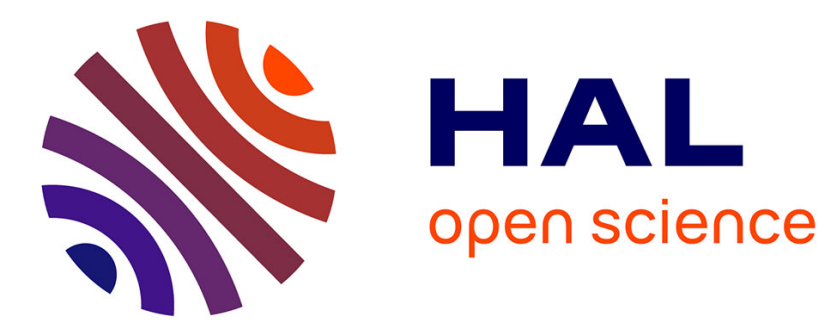

\title{
Study of Strain Effects on Carbon-Based Transistors With Semi-Analytic and Ab Initio Models
}

Yi Zheng, Fernando Zanella, Guido Valerio, Cesar Dartora, Zhuoxiang Ren

\section{To cite this version:}

Yi Zheng, Fernando Zanella, Guido Valerio, Cesar Dartora, Zhuoxiang Ren. Study of Strain Effects on Carbon-Based Transistors With Semi-Analytic and Ab Initio Models. IEEE Transactions on Magnetics, 2018, 54 (3), pp.1 - 4. 10.1109/TMAG.2017.2765705 . hal-01802421

\section{HAL Id: hal-01802421 \\ https: / hal.sorbonne-universite.fr/hal-01802421}

Submitted on 12 Mar 2020

HAL is a multi-disciplinary open access archive for the deposit and dissemination of scientific research documents, whether they are published or not. The documents may come from teaching and research institutions in France or abroad, or from public or private research centers.
L'archive ouverte pluridisciplinaire HAL, est destinée au dépôt et à la diffusion de documents scientifiques de niveau recherche, publiés ou non, émanant des établissements d'enseignement et de recherche français ou étrangers, des laboratoires publics ou privés. 


\title{
Study of Strain Effects on Carbon-Based Transistors with Semi-Analytic and Ab-Initio Models
}

\author{
Yi Zheng ${ }^{1}$, Fernando Zanella ${ }^{2}$, Guido Valerio ${ }^{1}$, Cesar A. Dartora ${ }^{2}$, and Zhuoxiang Ren ${ }^{1}$ \\ ${ }^{1}$ Sorbonne Universités, UPMC Univ. Paris 06, Paris 75005, France, yi.zheng@upmc.fr \\ ${ }^{2}$ Electrical Engineering Department, Federal University of Paraná (UFPR), Brazil, cadartora@eletrica.ufpr.br
}

\begin{abstract}
In this work, the current-voltage characteristics of a graphene-based transistor in the presence of external strain are computed with different methods. A simplified semi-analytic method allows fast computation of the electric parameters by granting a good accuracy. A more complex matrix method based on second quantized version of Schrodinger equation by means of non-equilibrium Green's function is used to validate the semi-analytic one. Both models are developed to evaluate the impact of mechanical stresses on this novel class of nano-transistors, where they are aimed for applications in the domain of flexible electronics.
\end{abstract}

Index Terms - Nanotransistors, graphene nanoribbon, deformation, non-equilibrium Green's function, flexible electronics.

\section{INTRODUCTION}

$\mathrm{C}$ arbon-based nanotransistors have recently attracted a lot $C_{\text {of attention due to their remarkable electronic properties }}$ [1]-[3]. As one of the emerging carbon materials, graphene has rapidly become an ideal candidate for flexible electronic devices [4]. In this connection, geometric deformation of graphene-based active components is an important issue.

Figs. 1 show an armchair graphene nanoribbon (aGNR), and an aGNR in a field-effect transistor (FET), where a strain is enforced on the FETs along the direction of the channel. To compute the drain/source current in the presence of this strain as a function of the voltages at the contacts, we propose two methods, having a different level of accuracy and complexity.

Both methods involve a multiscale coupling between a macro (the electrostatic behavior of the device) and a micromodel (the computation of the charge density in the channel). While the first model is formulated through Poisson equation, the second can be solved either with an approximated semianalytic solution or with a rigorous ab-initio approach.

The first choice requires the computation of an analytic expression for the aGNR energy band [5]. This approach was proposed in [6], and is here applied for the first time to study the effect of deformation, by using the analytical description of deformed aGNR presented in [7]. Interestingly, only small modifications to the original method are sufficient to obtain a complete semi-analytic model.

The second choice requires the numerical solution of Schrödinger equation in the Laplace domain, thus keeping a full information on the dynamic properties of the device. However, the current computation is done with a static approach, since only static characteristics of the transistors are computed [8]. Also in this case, a "deformed" Hamiltonian based on experimental data is proposed for the first time.

The general multiscale computational approach is discussed in Section II. The semi-analytical compact model is described in Section III, and the rigorous approach based on nonequilibriums Green's function (NEGF) is presented in Section IV. The two models will be used to study the impact of mechanical strains on the transistor properties in Section V.

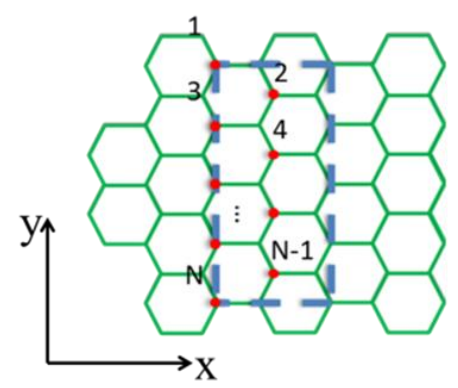

(a)

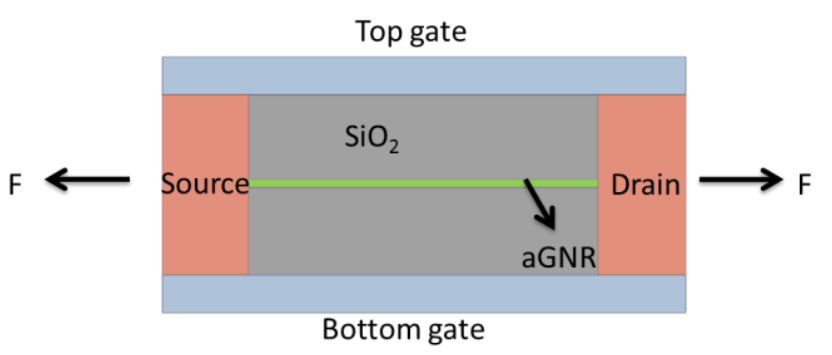

(b)

Fig. 1. (a) Armchair graphene nanoribbon. (b) Sketch of strain on double-gate aGNR FET.

\section{Computational APPROACH}

In this section, the multiscale coupling between the micromodel and the macro-model is discussed. As shown in Fig. 2, both models solve Poisson's equation in order to calculate the potential along the channel $\mu_{\mathrm{c}}$, starting from an initial guess value $\mu_{\mathrm{c} 0}$. The main difference is in the micro-model block, necessary to compute the charge density stored in the channel.

On one hand, in the semi-analytic micro-model we calculate analytical expressions for the energy bands of a graphene nanoribbon through a nearest-neighbor approach, which means that only the adjacent atoms interact with each other. This leads to closed form quantities (effective masses, densities of states), used to numerically calculate potentials and currents in the nano-device. On the other hand, the NEGF approach is a dynamical formulation based on the solution of Schrödinger equation, describing energy bands by using 
proper atomic orbitals for the coupling between carbon atoms, and between the source/drain and the graphene.

Once the charge is calculated with the relevant micromodel, the Poisson equation is solved again, and the loop is iterated until a convergence is obtained on the channel potential $\mu_{\mathrm{c}}$. Finally, the drain-source current $I_{\mathrm{ds}}$ is calculated (by using the potential $\mu_{\mathrm{c}}$ ) with a static model by considering the electron path inside the structure by means of creation and annihilation operators.

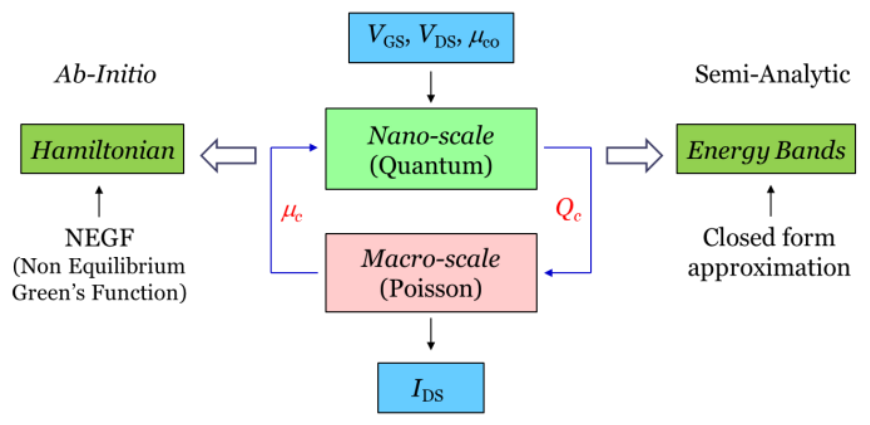

Fig. 2. Diagram used in both models to compute source-drain current.

\section{COMPACT MODEL}

In this section, we propose a semi-analytical model to study the effect of deformation in a graphene-nanoribbon transistor. In the absence of any deformation, graphene energy bands can be calculated with tight binding model [5].

$$
E_{\alpha}(k)= \pm V \sqrt{1+4 \cos \left(\frac{\sqrt{3} a k}{2}\right) A_{\alpha}+4 A_{\alpha}^{2}}
$$

where $A_{\alpha}=\cos \left(\frac{\pi \alpha}{N+1}\right) \cdot \alpha=1, \ldots, N$ defines the energy band, $a=2.46 \AA$, and $V=2.7 \mathrm{eV}$ is the tight-binding hopping energy without deformation. $k$, being the reciprocal of a length, is the momentum variable, directed in the nanoribbon direction.

Starting from (1), the effective mass, density of states and transmissivity coefficients $T_{s}$ and $T_{d}$ through the source and drain can be derived. These parameters are used to calculate the current by using the Landauer-Büttiker approach [8]:

$$
I_{i}\left(\mu_{c}\right)=\frac{q}{\pi \hbar} \sum_{\alpha} \int_{0}^{E_{\alpha}^{\max }-E_{\alpha}(0)} \frac{T_{s} T_{d}}{T^{\prime}}\left[f_{s}\left(\mu_{c}\right)-f_{d}\left(\mu_{c}\right)\right] \mathrm{d} E
$$

where $T^{\prime}=T_{s}+T_{d}-T_{s} T_{d}, q$ is the electronic charge, $f$ are Fermi-Dirac distributions of source and drain. The integration is performed over all the allowed energies in (1). The surface potential $\mu_{c}$ can be calculated by equating the microscopic charges $Q_{\mathrm{e} / \mathrm{h}}$ and the macroscopic charge $Q_{\text {macro }}$ [7]:

$$
Q_{\mathrm{h}}-Q_{\mathrm{e}}=Q_{\text {macro }}
$$

where

$$
\begin{gathered}
\frac{Q_{\mathrm{e} / \mathrm{h}}}{q}=\sum_{\alpha} \int_{0}^{E_{\alpha}^{\mathrm{max}}-E_{\alpha}(0)} D_{\alpha}(E)\left[\frac{T_{s}\left(2-T_{d}\right)}{T^{\prime}} f_{s}\left(\mu_{c}\right)+\right. \\
\left.+\frac{T_{d}\left(2-T_{s}\right)}{T^{\prime}} f_{d}\left(\mu_{c}\right)\right] \mathrm{d} E \\
Q_{\text {macro }}\left(\mu_{c}\right)=-\sum_{i=g, s, d} C_{i}\left(V_{i}-V_{F B, i}-\mu_{c}\right)
\end{gathered}
$$

and $C_{g}, C_{s}$ and $C_{d}$ are the capacitances of gate, source and drain, $V_{g}, V_{s}, V_{d}$ are the voltage of gate, source and drain, respectively, and $V_{\mathrm{FB}, i}$ are the relevant flat band voltages.

This approach can be simply modified in the presence of a relative deformation, or strain, $d(-0.1 \leq d \leq 0.1)$. $d$ is here the variation of distance between adjacent atoms normalized with respect to the non-deformed distance. In this case the hopping parameters change accordingly, thus determining a shift of Fermi points [7]. The energy bands of the graphene nanoribbon can be obtained:

$$
E_{\alpha}^{\mathrm{d}}(k) \cong \pm V \sqrt{1+4 \cos \left[(1+d) \frac{\sqrt{3} a k}{2}\right] A_{\alpha}^{\mathrm{d}}+4\left(A_{\alpha}^{\mathrm{d}}\right)^{2}}
$$

where

$$
\begin{gathered}
A_{\alpha}^{\mathrm{d}}=\cos \left(\frac{\pi \alpha}{N+1}-\Delta k_{y}\right) \quad \alpha=1, \ldots, N \\
\Delta k_{y}=-\frac{\sqrt{3}}{2} S_{t}(1+v) d
\end{gathered}
$$

$S_{\mathrm{t}}=1.2$ and $v$ is the Poisson's coefficient of the graphene, approximately equal to 0.145 [7].

Due to the importance of the energy gap value on the transistor performance, the impact of a uniaxial strain on the gap is further studied. The energy gap variation with respect to the strain is shown in Fig. 3.

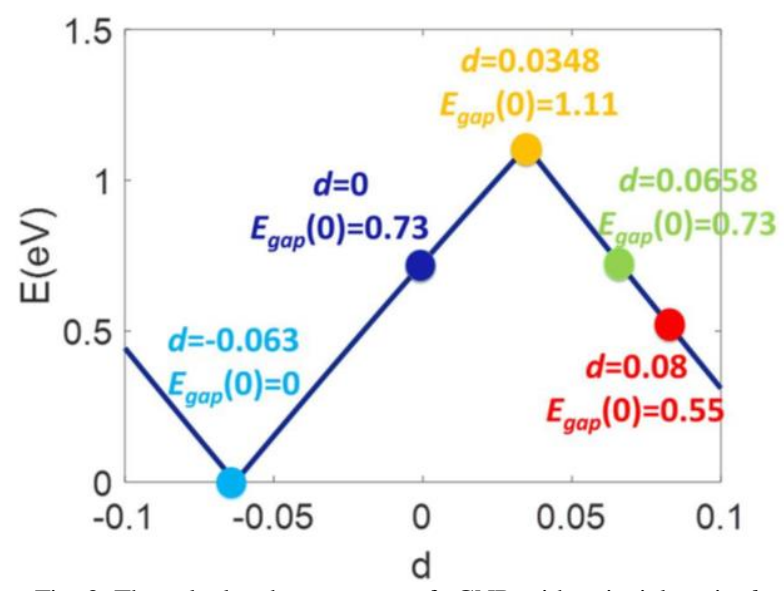

Fig. 3. The calculated energy gap of aGNR with uniaxial strain $d$. 


\section{NON EQUILIBRIUM GREEN'S FunCTION Method}

An extensive treatment of NEGF can be found in [8]-[9]; here we give a few definitions necessary to formulate our problem. The general form of the Green's function is given by:

$$
G_{i j}^{c}=-i S\left[c_{i}(t) c_{j}^{\dagger}\left(t^{\prime}\right)\right]
$$

where $c_{j}^{\dagger}\left(t^{\prime}\right)$ creates one electron at site $j$ at time $t^{\prime}$ and $c_{i}(t)$ annihilates an electron at site $i$ at time $t . S$ is a time-ordering operator that guarantees causality. Using expression (8) we can define the retarded Green's functions [9],

$$
G_{i j}^{r}\left(t, t^{\prime}\right)=-i \theta\left(t-t^{\prime}\right)\left\{c_{i}(t), c_{j}^{\dagger}\left(t^{\prime}\right)\right\}
$$

where $\theta(t)$ is the Heaviside function and $\{A, B\}=A B+B A$. Every atom in the lattice can be indexed by a couple of integers, and the interaction between sites by the indices $m n$, $i j$. To apply (9) to the nanoribbon, the equation of motion must be enforced:

$$
i \partial_{t} G_{m n, i j}^{r}\left(t, t^{\prime}\right)=\delta\left(t-t^{\prime}\right) \delta_{m n, i j}-i \theta\left(t-t^{\prime}\right)\left\{\left[c_{m n}, H\right], c_{i j}^{\dagger}\right\}
$$

All the physics will be described by the operator $H$ in (10):

$$
H_{i, j}=\frac{\hbar^{2}}{2 m_{e}} \nabla^{2}+\frac{q^{2} Z_{c}}{4 \pi \varepsilon_{0}}\left(\frac{1}{r_{i}-R_{j}+a_{0}}\right)+\mu_{c}
$$

where $q$ is the electron charge and $\hbar$ is the reduced Plank's constant. The first term in (11) is the kinetic energy of each electron, whit $m_{e}$ being the free electron mass. The second term is the Coulomb potential between an electron and a carbon nucleus, with $Z_{c}$ the effective atomic number and $\varepsilon_{0}$ the vacuum permittivity; $r_{i}-R_{j}$ is the distance between an electron and a nucleus, and $a_{0}$ is the maximum radius of a carbon atom, significant when $i=j$. The last term is the localized channel potential that must be found following the technique described in Section II.

By using (8) and path integrals properly defined by time ordering operators [9], it is possible to show that current can be calculated by the following equation

$$
\begin{gathered}
I_{\mathrm{ds}}\left(\mu_{c}\right)=\frac{-2 q}{\hbar} \int \operatorname{Tr}\left[\left(\boldsymbol{\Sigma}_{S}+\boldsymbol{\Sigma}_{S}^{\dagger}\right) \mathbf{G}^{r}\left(\mu_{c}\right)\left(\boldsymbol{\Sigma}_{D}+\boldsymbol{\Sigma}_{D}^{\dagger}\right) \mathbf{G}^{r}\left(\mu_{c}\right)^{\dagger}\right] \\
{\left[f_{S}\left(\mu_{c}\right)-f_{D}\left(\mu_{c}\right)\right] \mathrm{d} E}
\end{gathered}
$$

where $\boldsymbol{\Sigma}_{S}$ and $\boldsymbol{\Sigma}_{D}$ appearing in the trace of (12) capture the coupling between source/drain and graphene nanoribbon, and the symbol ${ }^{\dagger}$ stands for the Hermitian complex conjugate. By performing a Laplace transformation in (10) we obtain a matrix equation of the following form

$$
\boldsymbol{G}^{r}\left(E, \mu_{c}\right)=\left[E \mathbf{1}-i \eta \mathbf{1}-\boldsymbol{H}\left(\mu_{c}\right)-\boldsymbol{\Sigma}_{S}-\boldsymbol{\Sigma}_{D}\right]^{-1},
$$

where $E$ is the energy discretization and $\eta$ is an infinitesimal number necessary to guarantee convergence, and $\mathbf{1}$ is the unity matrix. $\boldsymbol{\Sigma}_{S, D}$ are obtained from (10) as

$$
\boldsymbol{\Sigma}_{S, D}=\boldsymbol{V}_{\mathrm{c}} \boldsymbol{g}_{S, D}^{r} \boldsymbol{V}_{c}^{\dagger}
$$

where $V_{\mathrm{c}}$ is a matrix where the elements are the coupling energies between channel and source/drain, $\boldsymbol{g}_{S, D}^{r}$ are the source/drain Green matrix, and is calculated also by (13) in the absence of the contacts, and with $\mu_{\mathrm{c}}=\mu_{\mathrm{S} / \mathrm{D}}$.

To find the matrix form of $\boldsymbol{H}\left(\mu_{c}\right)$ we project (11) in a $\pi$ orbital basis for the channel given by

$$
\varphi_{i, j}(r)=\sqrt{\frac{\lambda^{5}}{\pi}} \cos (\theta) e^{-\lambda r}
$$

where $\lambda=2.18$ is a constant enforcing orthonormality. For the contacts, we consider a coupling between a carbon atom and gold atom.

When a strain is applied, we use the elastic energy potential based on Hook's law, which is a Taylor expansion around the minimum of the energy bounding two atoms. This expansion, ignoring higher orders terms, has a parabolic form, where second derivative is known as the spring constant $K . K$ is found by considering the Poisson ratio and the graphene Young's module $Y$ by [10]

$$
K=\frac{1}{3} \cdot\left(\frac{Y}{1-2 v}\right)
$$

The Young's module is based on experimental data [11]. Also, the positions used in (11) must be modified, thus defining a "deformed" Hamiltonian.

\section{NUMERICAL RESULTS}

The same physical device is considered in both models. We plot in Fig. 4 the band diagram for the nanoribbon. Where dashed lines show the analytical approach used in (1), while solid lines come from the ab initio technic considering second nearest neighbors.

Despite the differences in the highest sub-bands, which for transport analyses are not important, we can see a very good match between the models. This result guarantees that the orbitals chosen for the ab-initio procedure described in the previous sections are capturing the correct physical effects, and that the near-neighbor approximation used in the semianalytic approach is sufficiently accurate.

In order to compute the drain-source current, we use the multiscale iterative coupling shown in Fig. 2. Since a ballistic regime is considered, the channel potential is assumed constant along the channel, so that the Poisson equation can be analytically solved. 


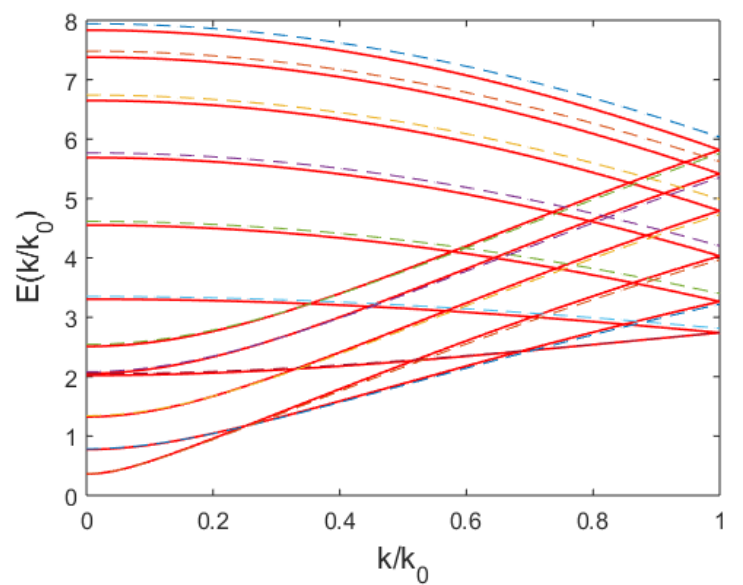

Fig. 4. Band diagram of aGNR without deformation. Ab-initio procedure (solid lines), analytic equation (dashed lines).

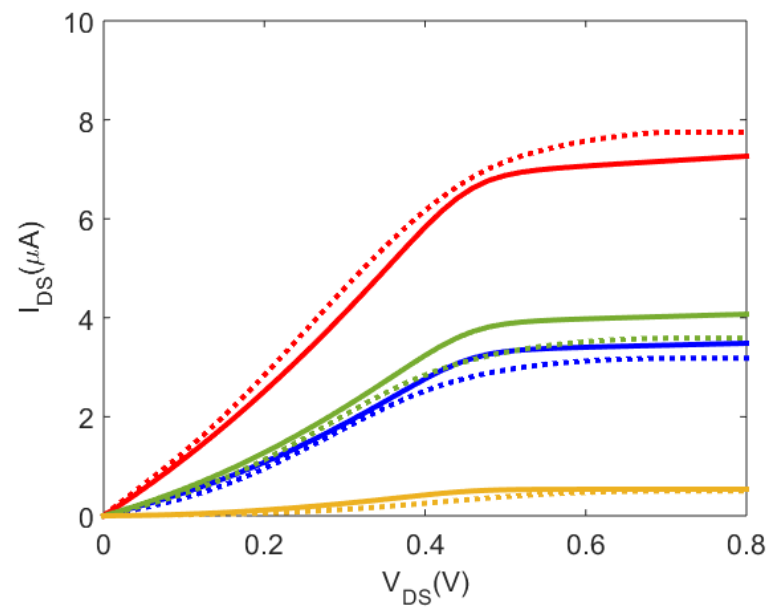

(a)

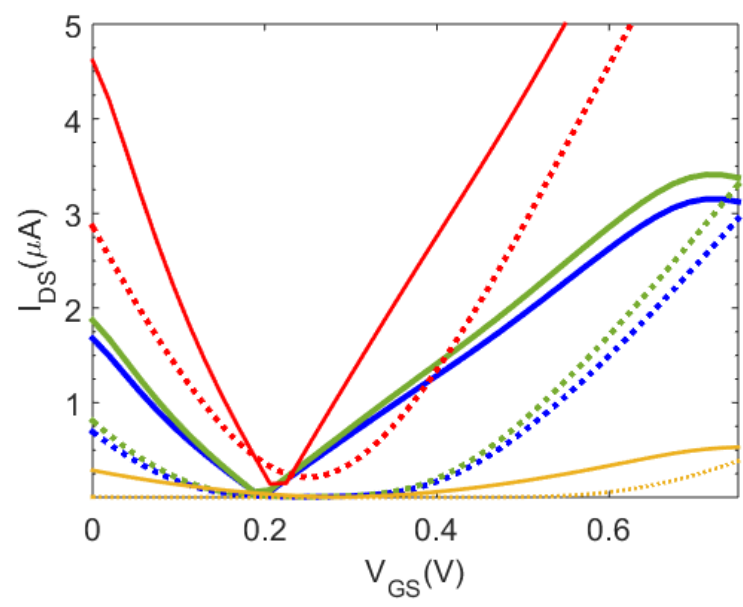

(b)

Fig. 5. (a) Current $I_{\mathrm{ds}}$ vs. drain-source voltage $V_{\mathrm{ds}} . V_{\mathrm{g}}=0.75 \mathrm{~V}$. (b) Current $I_{\mathrm{ds}}$ vs. drain-source voltage $V_{\mathrm{gs} .} V_{\mathrm{ds}}=0.5 \mathrm{~V}$.Semi-analytic (dashed lines) and $\mathrm{ab}$-initio results (solid lines). No deformation (blue lines), $d=0.0658$ (green lines), $d=0.08$ (red lines), $d=0.0348$ (yellow lines).
In Fig. 5 (a) the drain-source current $I_{\mathrm{ds}}$ is calculated by keeping a constant gate potential $V_{\mathrm{g}}=0.75 \mathrm{~V}$ and changing the drain-source potential. In Fig. 5 (b) a constant gate potential $V_{\mathrm{ds}}=0.5 \mathrm{~V}$ is kept and the drain-source potential varies. In both figures, dashed lines come from the semi-analytic procedure, while solid lines from the ab-initio technique. By varying the parameter $d$ the difference between the models is very small, meaning that the semi-analytic model correct captures the variation of the current with a reduced computational complexity.

Interestingly, the green case $(d=0.0658)$ has the same energy gap for the blue case $(d=0$, as shown in Fig. 3), but even so we can capture some differences in their current levels. This difference comes from the Schottky barrier present at the contacts in both models. This means that, even in the presence of the same energy gap, the semi-analytic model is able to capture the effects of a strain.

The semi-analytic method is by far faster than the ab-initio one. On an Intel Core i5-6300HQ @ 2.3GHzx4 and 8GiB of memory, the former requires around 1 minute of computation, while the latter takes more than 2 hours to compute 72 values of currents in the curves of Figs. 5.

\section{CONCLUSION}

A simple semi-analytical compact model has been compared with an accurate ab initio method based on NEGF, yielding very good agreement. The semi-analytic model captures the strain effects even in the presence of Schottky contacts, as proved by modeling the interaction between a carbon and gold atom in the ab-initio technique. Furthermore, the semi-analytic method being much faster than the ab-initio one, it can be used for CAD programs for the design of new architectures based on graphene where strain phenomena are relevant.

\section{REFERENCES}

[1] P. Avouris, J. Appenzeller, R. Martel, and S. Wind, "Carbon nanotube electronics," Proc. IEEE, vol. 91, no. 11, pp. 1772-1784, Nov. 2003.

[2] R. Martel, H. S. P. Wong, K. Chan, and P. Avouris, "Carbon nanotube field effect transistors for logic applications," in IEDM Tech. Dig., Washington, pp. 159-162, Dec. 2001.

[3] A. Javey, J. Guo, Q. Wang, M. Lundstrom, and H. Dai, "Ballistic carbon nanotube field-effect transistors," Nature, vol. 424, no. 6949, pp. 654657, Aug. 2003

[4] F. Schwierz, "Graphene transistors," Nature Nanotechnology, vol. 5, pp. 487-496, May. 2010.

[5] H. S. P. Wong and D. Akinwande, Carbon Nanotube and Graphene Device Physics. Cambridge University Press, 2011.

[6] G. Fiori and G. Iannaccone, "Simulation of graphene nanoribbon field effect transistors," IEEE Electron Devices Lett., vol. 28, no. 8, pp. 760762, Aug. 2007.

[7] Y. Li, X. Jiang, Z. Liu, Z. Liu, "Strain effects in graphene and graphene nanoribbons: the underlying mechanism," Nano Research, vol. 3, pp. 545-556, Jul. 2010.

[8] S. Datta. Electronic Transport in Electronic Systems. Cambridge University Press, Cambridge, 1995.

[9] P. C. Martin and J. Schwinger, "Conservation Laws and the Quantum Theory of Transport: The early days", Physical Review, vol. 115, pp 1342-1358, 1959.

[10] W. D. Callister and D. G. Rethwisch, Materials Science and Engineering. An Introduction. $8^{\text {th }}$ ed, Wiley, New York, Chapter 6.

[11] J.-U. Lee, D. Yoon and H. Cheong, "Estimation of Young's Modulus of Graphene by Raman Spectroscopy", Nano Lett., no. 12, vol. 9, pp 44444448, 2012. 